\title{
MATRIX REPRESENTATIONS OF SEMIGROUPS
}

\author{
by D. B. MCALISTER
}

(Received 24 August, 1965; and in revised form, 11 January, 1966)

In a series of papers [6], [7], [8], [10], Munn has considered the problem of constructing all irreducible representations of a semigroup by matrices over a field. In [10], he showed how to construct all the irreducible representations of an arbitrary inverse semigroup from those of associated Brandt semigroups. In this paper, we generalize the method of [10] to give a construction for the irreducible representations of an arbitrary semigroup from those of certain associated semigroups.

For many types of semigroups, including regular semigroups, periodic semigroups and 0 -simple semigroups with non-zero idempotents, the associated semigroups are completely 0 -simple. In this case, by means of Clifford's result [1] on the representations of a completely 0 -simple semigroup, we can give an explicit method of construction for all irreducible representations.

I should like to express my sincere gratitude to $\mathrm{Dr}$ W. D. Munn, who read the first rough draft of these results and who encouraged me to prepare them for publication.

1. $\mathscr{M}$-semigroups. In general, a semigroup need have neither a zero nor an identity. However, given any semigroup $S$, we may embed $S$ in a semigroup $S^{0}$ which has a zero and which is constructed from $S$ in the following way. If $S$ already has a zero and contains at least two members, then $S=S^{0}$; otherwise $S^{0}$ is the semigroup formed from $S$ by adjoining a new symbol 0 and defining $a 0=0=0 a$ for each $a \in S^{0}=S \cup\{0\}$. The phrase " $S=S^{0}$ " means that $S$ is a semigroup which has a zero and at least two members.

In a similar way, we can embed a semigroup $S$ in a semigroup $S^{1}$ that has an identity.

Because of the simple nature of the embedding of a semigroup $S$ in the corresponding semigroup $S^{0}$, many theorems about semigroups that have no zero may be deduced from corresponding theorems for semigroups that have a zero. In particular, there will be no loss of generality if, in this paper, we consider only semigroups that have a zero.

A homomorphism $\theta$ of a semigroup $S=S^{0}$ onto a semigroup $\bar{S}$ is said to be 0 -restricted if $a \theta=0 \theta$ implies $a=0$; the corresponding congruence on $S$ is also said to be 0 -restricted.

Proposition 1. Let $S=S^{0}$ be a semigroup. Then

$$
\rho=\left\{(a, b) \in S \times S: \text { for all } s, t \in S^{1}, \text { sat }=0 \text { if and only if } s b t=0\right\}
$$

is a 0 -restricted congruence on $S$. If $\tau$ is any 0 -restricted congruence on $S$, then $\tau \subseteq \rho$.

Proof. The relation $\rho$ is clearly an equivalence on $S$. Let $(a, b) \in \rho, x \in S$. Then, for any $s, t \in S^{1}$, sat $=0$ if and only if $s b t=0$. Hence, a fortiori, saxt $=0$ if and only if $s b x t=0$; thus $(a x, b x) \in \rho$. Similarly $(x a, x b) \in \rho$ and so $\rho$ is a congruence on $S$.

Let $a \in S$ with $(a, 0) \in \rho$. Then, for any $s, t \in S^{1}$, sat $=0$; in particular, $a=0$. Hence $(a, 0) \in \rho$ implies $a=0$ so that $\rho$ is a 0 -restricted congruence on $S$. 
Finally, let $\tau$ be any 0 -restricted congruence on $S$, and let $(a, b) \in \tau$. Then, by the regularity of $\tau$ with respect to multiplication, (sat, sbt) $\in \tau$ for all $s, t \in S^{1}$. Hence, in particular, for all $s, t \in S^{\mathbf{1}}$, sat $=0$ if and only if $s b t=0$. This means that $(a, b) \in \rho$; hence $\tau \subseteq \rho$.

The fact that $\rho$ is the maximum 0 -restricted congruence on $S$ may be deduced from the results of Preston [11] on subsets of a semigroup that are congruence classes. A proof is given here for completeness.

The congruence $\rho$ is of importance because, in many cases, a semigroup $S=S^{0}$ has an image of some particular type under a 0-restricted homomorphism if and only if $S / \rho$ is of that type. In particular, we have the following result.

PROPOSITION 2. Let $\mathscr{X}$ be a class of semigroups that is closed under homomorphic images. Then a semigroup $S=S^{0}$ has an image in $\mathscr{X}$ under a 0 -restricted homomorphism if and only if $S / \rho \in \mathscr{X}$.

Proof. If $S / \rho \in \mathscr{X}$, then $S$ has a 0-restricted homomorphic image in $\mathscr{X}$. Conversely, let $\tau$ be a 0 -restricted congruence on $S$ such that $S / \tau \in \mathscr{X}$. Then, since $\tau \subseteq \rho$, it follows, from the induced homomorphism theorem, that $S / \rho$ is a homomorphic image of $S / \tau$. Hence, by hypothesis, $S / \rho \in \mathscr{X}$.

Proposition 2 has several interesting corollaries. For example, let $S=S^{0}$ be a regular semigroup. Then, using Proposition 2, we can show that $S$ has an image under a 0 -restricted homomorphism that is an inverse semigroup if and only if, for any idempotents $e, f, g, h$ of $S$, $g e f h=0$ implies $g f e h=0$.

Munn [10] has shown that the following condition is important in the theory of matrix representations of a semigroup $S=S^{0}$.

$\mathrm{C}_{1}$ :

For any $a, x, b \in S$, if $a x b=0$, then $a x=0$ or $x b=0$.

He has also shown that the next condition plays an important part in the theory, if $S$ is an inverse semigroup.

$\mathrm{M}_{2}$ : If $M$ and $N$ are nonzero ideals of $S$, then $M \cap N \neq\{0\}$.

We shall see that, for arbitrary semigroups, condition

$\mathrm{C}_{2}$ : If $a, b \in S$ and $a S b=\{0\}$, then $a=0$ or $b=0$,

is more natural. The connection between $\mathrm{C}_{2}$ and $\mathrm{M}_{2}$ is given by the following proposition.

Proposition 3. Let $S=S^{0}$ be a semigroup. Then $S$ obeys $C_{2}$ if and only if it obeys $\mathrm{M}_{2}$ and

$\mathrm{C}_{2}^{\prime}$ : $\quad$ If $a \in S$ and $a S a=\{0\}$, then $a=0$.

Proof. Suppose first that $S$ obeys $\mathrm{C}_{2}$; then, clearly, $S$ obeys $\mathrm{C}_{2}^{\prime}$. Let $M$ and $N$ be nonzero ideals of $S$, and let $a, b$ be nonzero elements of $M$ and $N$ respectively. Then $a S b \subseteq M \cap N$ and, by $\mathrm{C}_{2}, a S b \neq\{0\}$. Hence $S$ obeys $\mathrm{M}_{2}$.

Conversely, suppose that $S$ obeys $\mathrm{M}_{2}$ and $\mathrm{C}_{2}^{\prime}$. Given nonzero ideals $M$ and $N$, let $a \in M \cap N \backslash\{0\}$. Then, by $\mathrm{C}_{2}^{\prime}$, axa $\neq 0$ for some $x \in S$ so that, since $a x a \in M . N, M . N \neq\{0\}$. 
In particular, given any nonzero elements $a, b \in S, S^{1} a S^{1} . S^{1} b S^{1} \neq\{0\}$. But

$$
S^{1} a S^{1} \cdot S^{1} b S^{1}=S^{1} a S b S^{1} \cup S^{1} a b S^{1},
$$

so that $a S b \neq\{0\}$ or $a b \neq 0$. If $a b \neq 0$, then, similarly, $a S a b \neq\{0\}$ or $a . a b \neq 0$. Thus, in any case, $a S b \neq\{0\}$. Hence $S$ obeys $C_{2}$. $\mathrm{M}_{2}$.

COROLlaRY. Let $S=S^{0}$ be a regular semigroup. Then $S$ obeys $C_{2}$ if and only if it obeys

We shall make use of Proposition 2 to give a short proof that $C_{1}$ and $C_{2}$ are necessary and sufficient for a semigroup $S=S^{0}$ to have a 0 -restricted congruence $\tau$ such that $S / \tau$ is completely 0 -simple. Since a completely 0-simple semigroup is regular, it follows from Theorem 1 of [9] and the corollary to Proposition 3 that these conditions are necessary. Another proof that $\mathrm{C}_{1}$ and $\mathrm{C}_{2}$ are both necessary and sufficient for the existence of a 0 restricted congruence $\tau$, with $S / \tau$ completely 0 -simple, has been given by Lallement [4].

A semigroup $S=S^{0}$ is said to be weakly regular if and only if, for each nonzero member $a$ of $S$, there exists $x \in S$ such that $a x=a x . a x \neq 0$.

Weakly regular semigroups have been called $E$-inversive, by Clifford and Preston [2], and 0 -inversive, by Lallement and Petrich [5].

The proof of the theorem makes use of the following result which is an immediate corollary to Theorem 3 of [5].

Proposition 4. Let $S=S^{0}$ be a semigroup that obeys $\mathrm{C}_{2}$. Then $S$ is completely 0-simple if and only if it is weakly regular and obeys the following weak cancellation law:

$\mathrm{C}_{3}: \quad$ If $a, b, x, y \in S$, then the relations $a x=b x \neq 0$ and $y a=y b \neq 0$

together imply that $a=b$.

THEOREM 1. Let $S=S^{0}$ be a semigroup that obeys $\mathrm{C}_{1}$. Then there is a 0-restricted congruence $\sigma$ on $S$ such that $S / \sigma$ obeys $\mathrm{C}_{3}$ and such that, if $\tau$ is any 0-restricted congruence on $S$ for which $S / \tau$ obeys $\mathrm{C}_{3}$, then $\sigma \subseteq \tau$.

Proof. We show first that $S / \rho$ obeys $C_{3}$. Let $a, b, x, y \in S$ be such that none of the elements $a x, b x, y a, y b$ is zero. Suppose, further, that $(a x, b x) \in \rho$ and $(y a, y b) \in \rho$. Then sat $=0$, for $s, t \in S^{1}$, implies $s a=0$ or $a t=0$. For, if $s, t \in S$, this is immediate from $C_{1}$ while, if, for example, $t \notin S$, then $s a t=s a$. If $s a=0$, then $s \in S$ and $s a x=0$; thus, since $(a x, b x) \in \rho$, $s b x=0$. Hence, by $\mathrm{C}_{1}$, since $b x \neq 0, s b=0$; thus $s b t=0$. Similarly, $a t=0$ implies $s b t=0$ and so $(a, b) \in \rho$. Thus $S / \rho$ obeys $\mathrm{C}_{3}$.

Let $T$ be the set of 0 -restricted congruences $\tau$ on $S$ such that $S / \tau$ obeys $C_{3} ; T \neq \square$ since $\rho \in T$. Let $\sigma=\bigcap\{\tau: \tau \in T\}$. Then it is immediate that $\sigma$ is a 0 -restricted congruence on $S$. It is also straightforward to verify that $S / \sigma$ obeys $C_{3}$. Thus, by its definition, $\sigma$ is the smallest 0 -restricted congruence $\tau$ on $S$ such that $S / \tau$ obeys $\mathrm{C}_{3}$.

COROLlary. Let $S=S^{0}$ be a semigroup. Then there is a 0-restricted congruence $\tau$ on $S$ such that $S / \tau$ is completely 0 -simple if and only if $S$ obeys $\mathrm{C}_{1}$ and $\mathrm{C}_{2}$. 
Proof. We have already pointed out that conditions $\mathrm{C}_{1}$ and $\mathrm{C}_{2}$ are necessary. To show that the conditions are sufficient, we need only show that $S / \rho$ is completely 0 -simple.

Let $a \in S \backslash\{0\}$; then, by $\mathrm{C}_{2}$, there exists $x \in S$ such that $a x a \neq 0$. If $s a t=0$ then, as in the proof of Theorem 1, either $s a=0$ or $a t=0$. In either case, saxat $=0$. Conversely, if saxat $=0$, then also saxa $=0$ or $a x a t=0$. Since $a x a \neq 0$, these imply respectively that $s a=0$ and $a t=0$; hence, in either case, sat $=0$. Thus $(a, a x a) \in \rho$ and so $S / \rho$ is regular.

Further, since $S$ obeys $\mathrm{C}_{2}$ and $\rho$ is a 0 -restricted congruence, it is easy to see that $S / \rho$ obeys $\mathrm{C}_{2}$. By the proof of Theorem $1, S / \rho$ obeys $\mathrm{C}_{3}$. Hence $S / \rho$ obeys the conditions of Proposition 4 and so is completely 0 -simple.

Let $S=S^{0}$ be a semigroup satisfying $\mathrm{C}_{1}$ and let $\sigma$ be the finest 0 -restricted congruence $\tau$ on $S$ such that $S / \tau$ obeys $\mathrm{C}_{3}$ (Theorem 1). Then we shall denote $S / \sigma$ by $S^{*}$.

Definition. A semigroup $S=S^{0}$ is called an $\mathscr{M}$-semigroup if it satisfies $\mathrm{C}_{1}$ and $\mathrm{C}_{2}$ and is such that $S^{*}$ is completely 0 -simple.

Proposition 5. Let $S=S^{0}$ be a weakly regular semigroup that obeys $\mathrm{C}_{1}$ and $\mathrm{C}_{2}$. Then $S$ is an $\mathscr{M}$-semigroup.

Proof. It is easy to verify that, if $\tau$ is any 0 -restricted congruence on $S$, then $S / \tau$ is weakly regular. In particular, since $S$ obeys $\mathrm{C}_{1}$ and $\mathrm{C}_{2}, S^{*}$ is weakly regular and obeys $\mathrm{C}_{2}$. Since $S^{*}$ obeys $\mathrm{C}_{3}$, it is thus immediate, from Proposition 4 , that $S^{*}$ is completely 0 -simple. Thus $S$ is an $\mathscr{H}$-semigroup.

COROLlaRY 1. Let $S=S^{0}$ be a periodic semigroup that satisfies $\mathrm{C}_{1}$ and $\mathrm{C}_{2}$; then $S$ is an $\mathscr{M}$-semigroup. In particular, any finite semigroup $S=S^{0}$ that satisfies $\mathrm{C}_{1}$ and $\mathrm{C}_{2}$ is an $\mathscr{M}$ semigroup.

Proof. Let $S$ be a periodic semigroup that obeys $\mathrm{C}_{1}$ and $\mathrm{C}_{2}$. Let $a \in S \backslash\{0\}$. By $\mathrm{C}_{2}$, there exists $x \in S$ such that $a x a \neq 0$. By induction on $n$, it follows from $\mathrm{C}_{1}$ that

$$
(a x)^{n}=a x . a x . \ldots . a x \neq 0
$$

for any positive integer $n$. Hence, for some positive integer $n,(a x)^{n}$ is a nonzero idempotent of $S$. Thus, since $(a x)^{n}=a \cdot(x a)^{n-1} x, S$ is weakly regular. Hence the result is immediate from Proposition 5.

COROllary 2. Let $S=S^{0}$ be a semigroup that satisfies $\mathrm{C}_{1}$ and $\mathrm{C}_{2}$ and that obeys the minimal conditions $\mathrm{M}_{L}$ and $\mathrm{M}_{R}$ on principal left and right ideals respectively. Then $S$ is an $\mathscr{M}$-semigroup.

Proof. Green [3, Theorem 4] has shown that $\mathrm{M}_{L}$ and $\mathrm{M}_{R}$ together imply the minimal condition $\mathrm{M}_{\boldsymbol{J}}$ on two-sided principal ideals. Hence $S$ has a 0 -minimal principal ideal $M$. Since $S$ obeys $\mathrm{C}_{2}, M^{2} \neq\{0\}$; thus $M$ is 0 -simple. Since $S$ obeys $\mathrm{M}_{L}$ and $\mathrm{M}_{R}, M$ must contain a 0 -minimal left ideal and a 0-minimal right ideal. Hence by [2, Corollary 2.50], $M$ is completely 0 -simple; thus it is regular. 
Let $a \in S \backslash\{0\}$ and let $x \in M \backslash\{0\}$. Then, by $\mathrm{C}_{2}$, there exists $y \in S$ such that ayx $\neq 0$. Since $x \in M$, so does ayx and hence, since $M$ is regular, there exists $z \in M$ such that

$$
a y z=a y x . z . a y x .
$$

Let $u=y x z$; then $a u$ is a nonzero idempotent of $S$. Hence $S$ is weakly regular.

Another important class of $\mathscr{M}$-semigroups is the class of all 0 -simple semigroups that obey $\mathrm{C}_{1}$ and which contain nonzero idempotents. For, suppose that $S=S^{0}$ is such a semigroup. Then $S^{*}$ is also 0 -simple and contains a nonzero idempotent. Now, if $e, f$ are nonzero idempotents of $S^{*}$, and $e f=f e \neq 0$, then

$$
e . e f=e f=e . f \neq 0 \text { and } e f . e=f e . e=f . e \neq 0 \text {. }
$$

Hence, by $\mathrm{C}_{3}, e f=f$; similarly, $f e=e$ so that $e=f$. Thus $S^{*}$ is a 0 -simple semigroup that contains a primitive idempotent. But, by $[2, \S 2.7]$, this means that $S^{*}$ is completely 0 -simple. Thus $S$ satisfies $\mathrm{C}_{2}$ and is an $\mathscr{M}$-semigroup.

In this paper, we shall determine each irreducible 0-restricted representation $\Gamma$ of an arbitrary semigroup $S=S^{0}$ modulo a representation of $M^{*}$, where $M$ is a certain ideal of $S$, dependent on $\Gamma$, which obeys $\mathrm{C}_{1}$ and $\mathrm{C}_{2}$. It follows that, if $M$ is an $\mathscr{M}$-semigroup, then the irreducible 0 -restricted representations of $S$ are known modulo those of completely 0 -simple semigroups and ultimately, by Clifford's result [1], modulo groups.

Munn [9] showed that, if $S=S^{0}$ is an inverse semigroup that obeys $\mathrm{C}_{1}$ and $\mathrm{M}_{2}$, then $S^{*} \cong M^{*}$ for any nonzero ideal $M$ of $S$. This does not hold in general; it need not even hold for an $\mathscr{M}$-semigroup, as the following simple example shows.

Example. Let $S=S^{0}$ be a completely 0-simple semigroup with no divisors of zero. Suppose further that $S$ is not a group with zero. Let $S^{1}$ be the semigroup formed by adjoining an identity to $S$. Then $S^{1}$ has no divisors of zero and so $S^{1}$ obeys $\mathrm{C}_{1}$ and $\mathrm{C}_{2}$.

Now $S$ is an ideal of $S^{1}$ and is completely 0 -simple, hence clearly $S^{*} \cong S$. On the other hand $S^{1}$ has an identity, so that $S^{1 *}$ is a group with zero.

If we consider the special case of weakly regular semigroups satisfying $C_{1}$ and $C_{2}$ and in which the idempotents commute, it can be shown that $S^{*}$ is a Brandt semigroup and that, in this case, there is an exact parallel with the results obtained by Munn [9], [10] for inverse semigroups. In particular, as for inverse semigroups, the finest 0 -restricted congruence $\sigma$ on $S$ such that $S / \sigma$ obeys $\mathrm{C}_{3}$ has the following simple form (cf. [9, Theorem 2.7]): for $a, b \in S$,

$$
(a, b) \in \sigma \text { if and only if } a=0=b \text { or } a x=b x \neq 0 \text { for some } x \in S \text {. }
$$

We end this section by giving a characterisation, for an arbitrary semigroup $S=S^{0}$ that obeys $\mathrm{C}_{1}$, of the 0 -restricted congruence $\sigma$ on $S$ whose properties were described in Theorem 1. The method of proof is similar to that used by Clifford [11] to describe the minimum cancellative congruence on a semigroup. As we do not need to make use of the construction, we omit the proof.

Let $S=S^{0}$ be a semigroup. Then, given any relation $\tau$ on $S$, we can construct new relations, from $\tau$, in the following ways. $\tau W=\left\{(a, b) \in S \times S:\right.$ for some $s, t \in S^{1},(a t, b t) \in \tau$ and $(s a, s b) \in \tau$, where none of $s a, s b, a t, b t$ is zero $\} \cup\{(0,0)\}$; 
$\tau C^{*}=\left\{(a, b) \in S \times S:\right.$ for some $s, t \in S^{1}, u, v \in S, a=s u t, b=s v t$ where $\left.(u, v) \in \tau\right\}$;

$\tau \circ \tau=\{(a, b) \in S \times S$ : for some $c \in S,(a, c) \in \tau,(c, b) \in \tau\}$;

$\tau \theta=\tau W \cup \tau C^{*} \cup(\tau \circ \tau)$ and $\tau \theta^{n}=\left(\tau \theta^{n-1}\right) \theta$.

If $\mathscr{I}$ is the identity congruence on $S$, we write $\mathscr{I} \theta^{n}=\theta^{n}$.

THEOREM 2. Let $S=S^{0}$ be a semigroup that obeys $\mathrm{C}_{1}$. Let $\tau$ be any 0-restricted congruence on $S$. Then the least congruence $\omega$ on $S$, containing $\tau$, such that $S / \omega$ obeys $C_{3}$ is $\tau \bar{\theta}=\bigcup_{n} \tau \theta^{n} ; \tau \bar{\theta}$ is a 0 -restricted congruence on $S$.

In particular, if $\sigma$ is the least 0 -restricted congruence $\omega$ on $S$ such that $S / \omega$ obeys $\mathrm{C}_{3}$, then $\sigma=\bar{\theta}=\bigcup_{n} \theta^{n}$. If, further, $S$ is an $\mathscr{M}$-semigroup, then $S / \sigma$ is the maximum completely 0 -simple 0 -restricted homomorphic image of $S$.

2. Representations over a field; introduction. Let $\Phi$ be a field, and let $n$ be a positive integer; then we denote by $(\Phi)_{n}$ the algebra of all $n \times n$ matrices over $\Phi$. The $n \times n$ identity is denoted by $I_{n}$.

A representation $\Gamma$ of a semigroup $S$, of degree $n$ over a field $\Phi$, is a homomorphism of $S$ into the multiplicative semigroup of $(\Phi)_{n}$. If $\Gamma$ is a representation of a semigroup $S=S^{0}$ of degree $n$ over a field $\Phi$ then, by convention, we consider $\Gamma(0)$ to be the $n \times n$ zero matrix, which we shall also denote by 0 . There is no loss of generality if we restrict $\Gamma$ in this way; see [10, pp. 167-168].

If $S$ is a semigroup, and $S \neq S^{0}$, then we may extend any representation $\Gamma$ of $S$ to a representation of $S^{0}$ by defining $\Gamma(0)$ to be the zero matrix. Consequently, it is sufficient to consider semigroups $S=S^{0}$.

Let $\Gamma$ be a representation of a semigroup $S=S^{0}$, of degree $n$ over a field $\Phi$. Then we define

$V(\Gamma)=\{x \in S: \Gamma(x)=0\} ;$

$r(\Gamma)=$ least positive integer $s$ such that, for some $x \in S, \Gamma(x)$ has rank $s$;

$M=M(\Gamma)=\{x \in S: \operatorname{rank} \Gamma(x) \leqq r(\Gamma)\}$, where $\operatorname{rank} \Gamma(x)$ is the usual matrix rank of $\Gamma(x)$.

$M(\Gamma)$ and $V(\Gamma)$ are clearly ideals of $S$, and there is a one-to-one correspondence between the representations $\Gamma$ of $S$ that vanish on an ideal $V$ (i.e. such that $V=V(\Gamma))$ and the 0 restricted representations of the Rees quotient semigroup $S / V$. (A representation $\Gamma$ of a semigroup $S=S^{0}$ is said to be 0-restricted if $\Gamma$ is a 0-restricted homomorphism.) It is thus sufficient to consider 0 -restricted representations of semigroups; this we do.

Munn $[10, \S 1]$, has essentially proved the following result.

LEMMA 1. Let $\Gamma$ be a 0 -restricted representation of a semigroup $S=S^{0}$. Then

(i) $M$ is an ideal of $S$ that obeys $\mathrm{C}_{1}$,

(ii) $\Gamma(M)$ obeys $\mathrm{C}_{3}$. 
A representation $\Gamma$ of a semigroup $S=S^{0}$, of degree $n$ over a field $\Phi$, is said to be irreducible if $\Gamma(S)$ is an irreducible matrix set, that is, if there is no fixed, nonsingular, $n \times n$ matrix $C$ such that, for each $x \in S$,

$$
C \Gamma(x) C^{-1} \text { has the block form }\left[\begin{array}{cc}
\Gamma_{1}(x) & 0 \\
A & \Gamma_{2}(x)
\end{array}\right] \text {, }
$$

where 0 denotes the zero $r \times(n-r)$ matrix, for some $1 \leqq r \leqq n$. Otherwise, $\Gamma$ is reducible.

Let $\Gamma$ be a representation of a semigroup $S=S^{0}$, of degree $n$ over a field $\Phi$, and let $T$ be a subset of $S$. Then we denote by $[\Gamma(T)]$ the subspace of $(\Phi)_{n}$ generated by $\Gamma(T)$. If $T$ is an ideal of $S$, then $[\Gamma(T)]$ is an ideal of the subalgebra $[\Gamma(S)]$ of $(\Phi)_{n}$. Further $\Gamma(T)$ is an irreducible matrix set if and only if the same is true of $[\Gamma(T)]$.

We now consider irreducible representations. The next two lemmas are classical; proofs may be found in [2, Chapter 5].

LEMMA 2. An irreducible subalgebra of $(\Phi)_{n}$ is a simple algebra over $\Phi$.

LEMMA 3. (Schur's Lemma) Let $\mathscr{A}$ be an irreducible subalgebra of $(\Phi)_{n}$. If $C$ is a constant nonzero matrix that commutes with each member of $\mathscr{A}$, then $C$ is nonsingular.

Using Lemmas 2, 3, Munn [7] proves the following result.

LemMa 4. Let $\Gamma$ be a 0 -restricted irreducible representation of $S=S^{0}$, of degree $n$ over a field $\Phi$. Let $\Gamma(T)$ be an irreducible subset of $\Gamma(S)$. Then there exist finite sets $e_{1}, \ldots, e_{r} \in T$, $\alpha_{1}, \ldots, \alpha_{r} \in \Phi$ such that

$$
\sum_{1}^{r} \alpha_{i} \Gamma\left(e_{i}\right)=I_{n}
$$

Lemma 5. Let $\Gamma$ be a 0 -restricted irreducible representation of $S=S^{0}$. Then $S$ obeys $\mathrm{C}_{2}$.

Proof. Let $a, b$ be nonzero elements of $S$; then $S^{1} a S^{1}, S^{1} b S^{1}$ are nonzero ideals of $S$. If $a S^{1} b=\{0\}$, then $S^{1} a S^{1} . S^{1} b S^{1}=\{0\}$; hence $\left[\Gamma\left(S^{1} a S^{1}\right)\right] .\left[\Gamma\left(S^{1} b S^{1}\right)\right]=\{0\}$.

By Lemma 2, $[\Gamma(S)]$ is a simple algebra; hence

$$
\left[\Gamma\left(S^{1} a S^{1}\right)\right]=[\Gamma(S)]=\left[\Gamma\left(S^{1} b S^{1}\right)\right] .
$$

Thus the hypothesis, $a S^{1} b=\{0\}$, implies that $[\Gamma(S)] \cdot[\Gamma(S)]=\{0\}$. But, by Lemma 4 , $I_{n} \in[\Gamma(S)]$, so this is impossible. Hence $a S^{1} b \neq\{0\}$; that is, $a S b \neq\{0\}$ or $a b \neq 0$. Suppose that $a b \neq 0$; then, as above, $a S^{1} a b \neq\{0\}$ and so $a S a b \neq\{0\}$ or $a . a b \neq 0$. In either case $a S b \neq\{0\}$; thus $S$ obeys $\mathrm{C}_{2}$.

3. Representations of a 0 -simple semigroup. Let $S=S^{0}$ be a 0 -simple semigroup, and let $\Gamma$ be a non-null representation of $S$, of degree $n$ over a field $\Phi$. Then, clearly, $\Gamma$ is a 0 -restricted representation and $M(\Gamma)=S$. Hence, by Lemma $1, S$ obeys $\mathrm{C}_{1}$. By means of a proof similar to that of Lemma 5 , we can show that any 0 -simple semigroup obeys $C_{2}$. Hence we have the following proposition, which may be used to give a sufficient condition for the existence of non-null representations of a 0 -simple semigroup; we shall consider this point in the next section. 
Proposition 6. Let $S=S^{0}$ be a 0-simple semigroup. Then $S$ obeys $\mathrm{C}_{2}$. Thus $S$ has a completely 0 -simple homomorphic image if and only if it obeys $\mathrm{C}_{1}$.

TheOREM 3. Let $S=S^{0}$ be a 0 -simple semigroup, and let $S$ obey $\mathrm{C}_{1}$. Let $S^{*}$ denote the maximum non-null homomorphic image of $S$ which obeys $\mathrm{C}_{3} ; S^{*}$ is clearly 0 -simple. Let $\Gamma$ be a non-null representation of $S$, of degree $n$ over a field $\Phi$. Then $\Gamma$ induces a non-null representation $\Gamma^{*}$ of $S^{*}$, of degree $n$ over $\Phi$, according to the rule: for each $\bar{x} \in S^{*}$,

$$
\Gamma^{*}(\bar{x})=\Gamma(x),
$$

where $x \rightarrow \bar{x}$ is the natural homomorphism of $S$ onto $S^{*}$.

Conversely, if $\Gamma^{*}$ is a non-null representation of $S^{*}$, of degree $n$ over $\Phi$, then the mapping $\Gamma$ of $S$ onto $\Gamma^{*}\left(S^{*}\right)$, defined by, for each $x \in S$,

$$
\Gamma(x)=\Gamma^{*}(\bar{x})
$$

is a non-null representation of $S$.

Proof. Since $S=M(\Gamma)=M, \Gamma(S)=\Gamma(M)$; hence, by Lemma $1, \Gamma(S)$ obeys $\mathrm{C}_{3}$. Thus $\Gamma(S)$ is a homomorphic image of $S^{*}$, and it follows, from the induced homomorphism theorem, that the mapping $\Gamma^{*}$ of $S^{*}$ onto $\Gamma(S)$, defined by (1), is a representation of $S^{*}$, of degree $n$ over $\Phi$.

The converse is immediate, since the composition of homomorphisms is a homomorphism.

COROLlaRY 1. Let $S=S^{0}$ be a 0 -simple $\mathscr{A}$-semigroup. Then the non-null representations of $S$ are those of its maximum completely 0-simple homomorphic image $S^{*}$.

Corollary 2. Let $S=S^{0}$ be a 0 -simple semigroup with identity. Then $S$ has a non-null representation if and only if it has no divisors of zero. In this case, the non-null representations of $S$ are those of its maximum group-with-zero homomorphic image $S^{*}$.

Proof. Suppose that $\Gamma$ is a non-null representation of $S$. Then $S$ obeys $\mathrm{C}_{1}$, and is an $\mathscr{M}$-semigroup. Thus $S^{*}$ is a completely 0 -simple semigroup with identity; that is, $S^{*}$ is a group-with-zero. Hence $S$ has no divisors of zero. The remainder of the result is now immediate from Corollary 1.

Clifford [1] has given a construction for all non-null representations of a completely 0 -simple semigroup. Taken with Corollary 1 and Theorem 3, this provides a construction for all representations of a 0 -simple $\mathscr{M}$-semigroup. It should be noted however that not every 0 -simple semigroup is an $\mathscr{U}$-semigroup. For example, let $S$ be the multiplicative semigroup of all $2 \times 2$ matrices over the reals, of the form

$$
\left[\begin{array}{ll}
a & 0 \\
b & 1
\end{array}\right],
$$

where $a$ and $b$ are positive real numbers; then $S$ is a simple cancellative semigroup [2, Chapter $5, \S 5$, Example $7(\mathrm{~b})]$. Thus $S^{0}$ is a 0 -simple semigroup that obeys $\mathrm{C}_{1}$ and $\mathrm{C}_{3}$. But $S^{0}$ has no nonzero idempotents and so is not completely 0 -simple. 
Theorem 3 shows that, for any 0-simple semigroup $S=S^{0}$, there is a one-to-one correspondence between the representations of $S$ and those of $S^{*}$. It is an easy matter to prove that this correspondence preserves equivalence, decomposition and reduction of representations. For the definitions of equivalence and decomposition of representations, see, for example, [2, Chapter 5].

4. Irreducible representations of an arbitrary semigroup. The main result of this section gives a method of construction for all 0-restricted irreducible representations of an arbitrary semigroup $S=S^{0}$, from those of certain associated semigroups. By Lemma 5 , if such a representation exists, then $S$ satisfies $\mathrm{C}_{2}$ and, by Lemma 6 below, so also does any nonzero ideal of $S$. Further, if $S$ has the property that each nonzero ideal of $S$ that satisfies $C_{1}$ is an $\mathscr{M}$-semigroup, then each of these associated semigroups is completely 0 -simple. In this case, we have an explicit construction for the irreducible 0 -restricted representations of $S$.

THEOREM 4. Let $S=S^{0}$ be a semigroup which obeys $\mathrm{C}_{2}$. Let $\mathrm{\Gamma}$ be a 0 -restricted irreducible representation of $S$, of degree $n$ over a field $\Phi$. Then $\Gamma$ induces a 0 -restricted irreducible representation $\Gamma^{*}$ of $M^{*}$, where $M=M(\Gamma)$, and there are finite sets of elements $e_{1}, \ldots, e_{r} \in M$, $\alpha_{1}, \ldots, \alpha_{r} \in \Phi$ such that, for each $x \in S$,

$$
\Gamma(x)=\sum_{1}^{r} \alpha_{i} \Gamma^{*}\left(\overline{e_{i} x}\right),
$$

where $x \rightarrow \bar{x}$ is the natural homomorphism $M \rightarrow M^{*}$.

Conversely, let $M$ be a nonzero ideal of $S$ that obeys $\mathrm{C}_{1}$, and let $\Gamma^{*}$ be a 0 -restricted irreducible representation of $M^{*}$, of degree $n$ over $\Phi$. Then, for any finite sets $e_{1}, \ldots, e_{r} \in M$, $\alpha_{1}, \ldots, \alpha_{r} \in \Phi$ such that

$$
\sum_{1}^{r} \alpha_{i} \Gamma^{*}\left(\bar{e}_{i}\right)=I_{n},
$$

the mapping $\Gamma$ of $S$ into $(\Phi)_{n}$, defined by (2), is a 0 -restricted irreducible representation of $S$, of degree $n$ over $\Phi$. The representation is independent of the particular choice of elements $e_{i}, \alpha_{i}$ satisfying (3).

Let $\Gamma_{1}$ and $\Gamma_{2}$ be 0-restricted irreducible representations of $S=S^{0}$, defined, as above, from ideals $M_{1}$ and $M_{2}$ of $S$. Then $\Gamma_{1}$ and $\Gamma_{2}$ are equivalent if and only if they are equivalent on $M_{1} \cap M_{2}$.

Proof. Let $M$ and $\Gamma$ satisfy the hypothesis of the first part of the theorem. By Lemma 1, $\Gamma(M)$ obeys $\mathrm{C}_{3}$. Hence the mapping $\Gamma^{*}$ defined by the rule

$$
\Gamma^{*}(\bar{x})=\Gamma(x),
$$

for each $\bar{x} \in M^{*}$, where $x \rightarrow \bar{x}$ is the natural homomorphism of $M$ onto $M^{*}$, is a 0 -restricted 
representation of $M^{*}$ over $\Phi$, of the same degree as $\Gamma$. Since $M$ is an ideal of $S$, and $\Gamma(S)$ is an irreducible matrix set, it follows from Lemma 2 that

$$
\left[\Gamma^{*}\left(M^{*}\right)\right]=[\Gamma(M)]=[\Gamma(S)] .
$$

Hence $\Gamma^{*}$ is an irreducible representation of $M^{*}$.

From Lemma 4 , since $\Gamma^{*}$ is irreducible, there exist $\bar{e}_{1}, \ldots, \bar{e}_{r} \in M^{*}$ and $\alpha_{1}, \ldots, \alpha_{r} \in \Phi$ such that

$$
\sum_{1}^{r} \alpha_{i} \Gamma^{*}\left(\bar{e}_{i}\right)=I_{n} .
$$

Choose $e_{i} \in M$ such that $e_{i} \rightarrow \bar{e}_{i}$ for each $1 \leqq i \leqq r$. Then, for each $x \in S$,

$$
\Gamma(x)=I_{n} \Gamma(x)=\left(\sum_{1}^{r} \alpha_{i} \Gamma^{*}\left(\tilde{e}_{i}\right)\right) \cdot \Gamma(x) .
$$

But $\Gamma^{*}\left(\bar{e}_{i}\right)=\Gamma\left(e_{i}\right)$ for each $1 \leqq i \leqq r$; hence, since $M$ is an ideal of $S$,

$$
\Gamma(x)=\sum_{1}^{r} \alpha_{i} \Gamma\left(e_{i}\right) \Gamma(x)=\sum_{1}^{r} \alpha_{i} \Gamma\left(e_{i} x\right)=\sum_{1}^{r} \alpha_{i} \Gamma^{*}\left(\overline{e_{i} x}\right) .
$$

This completes the proof of the first part.

The proof of the converse follows exactly as in the case of principal irreducible representations; cf. [7, Theorem 1].

Finally, it is clear that the criterion for equivalence is necessary. Suppose that $\Gamma_{1}$ and $\Gamma_{2}$ are equivalent on $M_{1} \cap M_{2}$. By $\mathrm{C}_{2}, M_{1} \cap M_{2}$ is a nonzero ideal of $S$ and hence

$$
\left[\Gamma_{1}\left(M_{1} \cap M_{2}\right)\right]
$$

is a nonzero ideal of $\Gamma_{1}(S)$. But, by Lemma 2, this means that $\left[\Gamma_{1}\left(M_{1} \cap M_{2}\right)\right]=\left[\Gamma_{1}(S)\right]$. Thus, by Lemma 4, we can choose $e_{1}, \ldots, e_{r} \in M_{1} \cap M_{2}$ and $\alpha_{1}, \ldots, \alpha_{r} \in \Phi$ such that

$$
\sum_{1}^{r} \alpha_{i} \Gamma_{1}\left(e_{i}\right)=I_{n}
$$

Since $\Gamma_{1}$ and $\Gamma_{2}$ are equivalent on $M_{1} \cap M_{2}$, there exists a nonsingular matrix $A$ such that, for each $m \in M_{1} \cap M_{2}$,

$$
\Gamma_{2}(m)=A \Gamma_{1}(m) A^{-1}
$$

Hence $\sum_{1}^{r} \alpha_{i} \Gamma_{2}\left(e_{i}\right)=I_{n} ;$ thus, for each $x \in S$,

$$
\Gamma_{2}(x)=\sum_{1}^{r} \alpha_{i} \Gamma_{2}\left(e_{i} x\right)=\sum_{1}^{r} \alpha_{i} A \Gamma_{1}\left(e_{i} x\right) A^{-1}=A \Gamma_{1}(x) A^{-1} .
$$

That is, $\Gamma_{1}$ and $\Gamma_{2}$ are equivalent. 
Note 1 . It can readily be shown that, if, in the above theorem, $S$ is a regular semigroup, a periodic semigroup, a semigroup satisfying $\mathrm{M}_{L}$ and $\mathrm{M}_{R}$, or a 0 -simple semigroup containing a nonzero idempotent, then $M$ is an $\mathscr{M}$-semigroup (note Lemma 6); that is, $M^{*}$ is completely 0 -simple. In this case the 0-restricted irreducible representations of $S$ can be determined explicitly by means of Clifford's theory of representations of a completely 0 -simple semigroup [1].

Note 2. Let $S=S^{0}$ be a semigroup satisfying $\mathrm{C}_{2}$ that has a unique minimal nonzero ideal. Then, by the last part of Theorem 4, the irreducible 0 -restricted representations of $S$ are determined, to within equivalence, by those of the unique minimal nonzero of $S$. That is, in the terminology of [7], they are the principal irreducible 0-restricted representations of $S$.

We shall end the paper by giving a sufficient condition for the existence of a 0 -restricted representation of a semigroup $S=S^{0}$, that obeys $C_{2}$. Before giving this criterion, we shall prove some results about conditions $C_{1}$ and $C_{2}$.

Lemma 6. Let $S=S^{0}$ be a semigroup that obeys $\mathrm{C}_{2}$. Let $L$ be a nonzero ideal of $S$. Then L obeys $\mathrm{C}_{2}$.

Proof. Let $m, n$ be nonzero members of $L$. Then, by $\mathrm{C}_{2}$, there exists $x \in S$ such that $m x m \neq 0$. Again, by $\mathrm{C}_{2}$, there exists $y \in S$ such that $m x m \cdot y . n \neq 0$. Let $u=x m y$; since $L$ is an ideal of $S, u \in L$. Then $m u n \neq 0$ and so $L$ obeys $\mathrm{C}_{2}$.

Lemma 7. Let $S=S^{0}$ be a semigroup that obeys $\mathrm{C}_{2}$. Then the set of all ideals of $S$ that obey $\mathrm{C}_{1}$ has a unique maximal member $L$.

Proof. Let $L=\bigcup\left\{L_{\alpha}: \alpha \in A\right\}$ be the union of all ideals of $S$ that obey $C_{1}$. If $L \neq\{0\}$, let $a \in L \backslash\{0\}$, and suppose that $s a \neq 0$ and $a t \neq 0$ for $s, t \in S$; then $a \in L_{\alpha}$, for some $\alpha \in A$. Since, by Lemma $6, L_{\alpha}$ obeys $C_{2}$, there exist $m, n \in L_{\alpha}$ such that $m s a \neq 0$, at $\neq 0$. Since $L_{\alpha}$ is an ideal that obeys $\mathrm{C}_{1}$, it follows that msatn $\neq 0$; hence sat $\neq 0$. Thus $L$ obeys $\mathrm{C}_{1}$.

THeOREM 5. Let $S=S^{0}$ be a semigroup that obeys $\mathrm{C}_{1}$ and $\mathrm{C}_{2}$, and let $T$ be a nonzero ideal of $S$. If $\sigma$ and $\tau$ denote, respectively, the maximum 0 -restricted congruences on $S$ and $T$, then

$$
S / \sigma \cong T / \tau \text {. }
$$

Proof. Since $S$ obeys $\mathrm{C}_{1}$ and $\mathrm{C}_{2}$, it follows, from Lemma 6, that the same is true of $T$. From the definitions of $\sigma$ and $\tau$, it is clear that, for $a, b \in T$, if $(a, b) \in \sigma$ then $(a, b) \in \tau$. Conversely, let $(a, b) \in \tau$ and let $s a t=0$, where $s, t \in S^{i} \backslash 0$. Since $T$ is an ideal of $S$ and $S$ obeys $C_{2}$, there exist $m, n \in T$ such that neither of $m s, t n$ is zero. Then $m s a t n=0$ and so, since $(a, b) \in \tau$, $m s b t n=0$. Since $S$ obeys $C_{1}, m s b t n=0$ implies $m s b t=0$ or $s b t n=0$. But neither of $m s, t n$ is zero so that each of these equations implies $s b t=0$. Similarly, $s b t=0$ implies $s a t=0$; hence $(a, b) \in \sigma$. Thus $\tau=\sigma \cap(T \times T)$.

Let $\theta$ denote the natural homomorphism of $S$ onto $S / \sigma$. Then, since $\tau=\sigma \cap(T \times T)$,

$$
T / \tau \cong T \theta \text {. }
$$

But, by the proof of the corollary to Theorem $1, S \theta=S / \sigma$ is completely 0 -simple. Thus, since $T$ is a nonzero ideal of $S, T \theta=S \theta$. Hence we have the result. 
Let $S=S^{0}$ be a semigroup that obeys $\mathrm{C}_{2}$, and let $M$ be an ideal of $S$ that obeys $\mathrm{C}_{1}$. Then a sufficient condition for $S$ to have a 0 -restricted representation, over a field $\Phi$, defined from $M$ as in Theorem 4, is that $M / \rho$ should have a 0 -restricted irreducible representation over $\Phi$. In fact, by Theorem 5 , it is sufficient that $L / \rho$ should have a 0 -restricted irreducible representation over $\Phi$. By the proof of the corollary to Theorem $1, L / \rho$ is completely 0 -simple; hence we can use Clifford's results [1] to give necessary and sufficient conditions for $L / \rho$ to have an irreducible 0-restricted representation.

Clifford proves the following. Let $\mathfrak{n}^{0}(G ; I, \Lambda ; P)$ be a regular Rees matrix semigroup over a group with zero $G^{0}$. Let $\Gamma$ be a representation of $G$ of degree $n$ over a field $\Phi$. Then $\Gamma$ can be extended to a representation of $\mathfrak{n}^{0}(G ; I, \Lambda ; P)$ if and only if the $\Lambda \times I$ block matrix $\Omega$ over $\Phi$, whose $(\lambda, i)$ th block is the $n \times n$ matrix $\Gamma\left(p_{\lambda i}\right)-\Gamma\left(p_{\lambda 1} p_{1 i}\right)$, has finite rank over $\Phi$. Further, every representation of $\mathfrak{m}^{0}(G ; I, \Lambda ; P)$ is the extension of some representation of $G$; in particular, the irreducible representations are the extensions of irreducible representations of $G$.

Let $a \in L \backslash\{0\}$; if $a^{2} \neq 0$, then (cf. the proof of the corollary to Theorem 1) $\left(a, a^{3}\right) \in \rho$ and $\left(a, a^{6}\right) \in \rho$, so that $\left(a, a^{2}\right) \in \rho$. Thus $L / \rho$ is a completely 0 -simple semigroup in which each element is either idempotent or nilpotent. Hence [2] $L / \rho$ is isomorphic to a regular Rees matrix semigroup over a group-with-zero $G^{0}$; further, since each element of $L / \rho$ is either idempotent or nilpotent, it can be verified by direct calculation that $G$ has only one element.

Suppose that $L / \rho \cong \mathfrak{n}^{0}(\{e\} ; I, \Lambda ; P)$, where $\{e\}$ is a one element group. Let $\Phi$ be a field and let $\Omega$ be the $\Lambda \times I$ matrix over $\Phi$ where $\Omega_{\lambda i}=1,0,-1$ according as $p_{\lambda i}$ is greater than, is equal to, is less than $p_{\lambda 1} p_{1 i} ;\{e\}^{0}$ is partially ordered by $e>0$. If $\Omega$ has finite rank over $\Phi$, then we say that $S$ has finite rank over $\Phi$; if $L=\{0\}$, then rank $S$ is zero.

Since $\{e\}$ has only one member, every irreducible representation of $\{e\}$ over $\Phi$ is of degree one. Hence, by Clifford's results, mentioned above, $L / \rho$ has an irreducible representation over $\Phi$ if and only if $\Omega$ has finite rank.

The above results are gathered together in the following proposition.

Proposition 7. Let $S=S^{0}$ be a semigroup that obeys $\mathrm{C}_{2}$, and let $\Phi$ be a field. If $S$ has a 0 -restricted representation over $\Phi$, then $S$ has nonzero rank over $\Phi$. Conversely, if $S$ has finite nonzero rank over $\Phi$, then $S$ has a 0 -restricted representation over $\Phi$.

Finally, we point out that, if $S=S^{0}$ is an inverse semigroup or a weakly regular semigroup in which the idempotents commute, it can be shown that the criterion of Proposition 7 is not only sufficient but is also necessary; cf. [10] for the inverse case. In this case it takes the form: $S$ has a 0 -restricted representation if and only if $L / \rho$ is finite with at least two members.

\section{REFERENCES}

1. A. H. Clifford, Matrix representations of completely 0-simple semigroups, American $J$. Math. 64 (1942), 327-342.

2. A. H. Clifford and G. B. Preston, Algebraic theory of semigroups, Vol. 1, American Math. Soc. Surveys, 7 (1961).

3. J. A. Green, On the structure of semigroups, Ann. of Math. 54 (1951), 163-172.

4. G. Lallement, Sur les homomorphismes d'un demigroupe sur un demigroupe completement 0-simple, Seminaire Dubreil-Pisot, 1963-64, no. 14. 
5. G. Lallement and M. Petrich, Some results concerning completely 0-simple semigroups, Bull. Amer. Math. Soc. 70 (1964), 777-778.

6. W. D. Munn, Matrix representations of semigroups, Proc. Cambridge Phil. Soc. 53 (1957),

7. W. D. Munn, Irreducible matrix representations of semigroups, Quart. J. Math. (Oxford Ser.) (2) 11 (1960), 295-309.

8. W. D. Munn, A class of irreducible matrix representations of an arbitrary inverse semigroup, Proc. Glasgow Math. Assoc. 5 (1961), 41-48.

9. W. D. Munn, Brandt congruences on inverse semigroups, Proc. London Math. Soc. (3) 14 (1964), 154-164.

10. W. D. Munn, Matrix representations of inverse semigroups, Proc. London Math. Soc. (3) 14 (1964), 165-181.

11. G. B. Preston, Congruences on semigroups (Ed. J. M. Howie), N.S.F. Algebra Institute. Pennsylvania State University, Summer 1963.

Department of Pure Mathematics

QUEEN'S UNIVERSITY

BELFAST 